\title{
Interception of a Projectile Using a Human Vision-Based Strategy
}

\author{
Justin A. Borgstadt and Nicola J. Ferrier* \\ Mechanical Engineering, University of Wisconsin-Madison \\ Madison, WI, USA 53706 \\ justin@mechatron.me.wisc.edu ferrier@mechatron.me.wisc.edu
}

\begin{abstract}
Visual-servoing tasks for mobile robots characteristically require the processing of vast amounts of navigational information which can impede the performance of even the most well designed systems. This paper investigates the idea of using a simple human-based strategy to guide a mobile robot to intercept a projectile. This vision based control strategy relies on a single image-based parameter whose sign corresponds to a decision whether to run forward or backward for successful interception. Assuming the ball can be tracked easily in real time by the robot's vision system, motion decision making and control computations are minimized. We examine and develop several motion control strategies which incorporate the robot's velocity and acceleration in addition to this human-based strategy. The simplest strategy is tested on a mobile robot and demonstrates results similar to a human.
\end{abstract}

\section{Introduction}

Much effort has been given to the design of robust robotic systems which can perform complicated tasks quickly and efficiently. One method of design involves extensive 3-D dynamic modeling of both the workspace and robot. While this approach has been proven to be effective in real-time for even difficult tasks [1] the cost in terms of required hardware, computational power, and design time can be extensive. McLeod and Dienes present an empirical model based on how humans intercept balls projected towards them [3]. Their basic assumption is that the fielder cannot possibly know exactly where the ball will land by calculating velocity, projection angle, wind resistance, and other factors affecting the flight path in the time allowed. However, human fielders do catch balls very effectively. They proposed that a fielder "unconsciously" learns to run "at a speed which keeps

\footnotetext{
*This research supported in part by NSF IRI-9703352
}

$d^{2}(\tan \alpha) / d t^{2}$ zero, where $\alpha$ is the vertical angle of gaze as they watch the ball". Hence, the fielder arrives at the point where the ball will land at the exact same time the ball arrives [3]. Videotaped evidence of several different human fielders catching balls of varying projectile motions supports the idea that the sign of $d^{2}(\tan \alpha) / d t^{2}$ may be used as an input to a mechanism designed to catch a projectile indicating whether to move forward, backward, or stay stationary.

The sign of $d^{2}(\tan \alpha) / d t^{2}$ may tell the fielder which direction to move but the fielder's velocity and acceleration are also needed in a control structure. The first part of this paper describes the details of how the robotic fielder tracks the ball and calculates the motion parameter $d^{2}(\tan \alpha) / d t^{2}$. Then four different motion control strategies are discussed. Simulations determine which strategy best utilizes the sign of $d^{2}(\tan \alpha) / d t^{2}$ to allow a robotic fielder to successfully intercept projected balls. The last part of this paper deals with applying a successful simulated motion control strategy to a real world situation. A ball is projected in real-time towards a mobile robot equipped with a vision system to demonstrate the feasibility of using the human-based model for visual-servoing.

\section{Control Parameter}

In order to accurately compute the motion parameter $d^{2}(\tan \alpha) / d t^{2}$ three things need to be achieved: image based tracking of the projectile, projectile fixation, and error minimization in the computation of the discrete variables $\tan \alpha$ and $d(\tan \alpha) / d t$ between successive frames. This investigation used an RWI B21 Mobile Robot, Directed Perception pan-tilt unit and a Sony CCD camera.

Image based tracking of the projectile was achieved by using a white ball on a dark background. Grey-scale images were thresholded and the centroid of the ball computed. The ball was assumed to be traveling in a purely vertical direction with respect to the robot 
which enabled a reduction in the computational time by processing a narrow sub-image window.

In order to fixate on the projectile and to keep the projectile in the field of view, a pan-tilt unit was used move the camera. The projection of the centroid was kept along the camera frame z-axis (i.e. at the center of the image, Fig. 1). The tilt angle $\beta_{n}$ was the angle required to bring the centroid of the projectile to the center of the image in the $n^{\text {th }}$ frame and thus follow the ball. $\beta_{n}$ was computed for each frame using the classical pinhole model, $\beta_{n}=\tan ^{-1}[(0.5 h-v) / f]$. For the $n^{t h}$ frame the gaze angle, $\alpha_{n}$, is computed as the previous gaze angle incremented by $\beta_{n}$, i.e. $\alpha_{n}=\alpha_{n-1}+\beta_{n}$, with $\alpha_{0}=0$ (Fig. 1) .

With three values for the gaze angle, $\alpha_{n}, \alpha_{n-1}$, and $\alpha_{n-2}$, and their corresponding tangent values, the discrete-motion parameter $d^{2}\left(\tan \alpha_{n}\right) / d t^{2}$ is computed by second difference methods [4]. Two factors contribute to errors in the calculation of $d^{2}\left(\tan \alpha_{n}\right) / d t^{2}$. First, $d^{2}\left(\tan \alpha_{n}\right) / d t^{2}$ is at best an an average value over a time period of $2 d t$. Second, the robot frame of reference from which $\alpha_{n}$ is being measured is moving relative to the projectile at a variable rate. The higher the relative velocity between the robot and projectile the more error will be incurred in the successive values of $\alpha_{n}$ and, therefore, $d^{2}\left(\tan \alpha_{n}\right) / d t^{2}$. A way to decrease both effects is to decrease the value of $d t$. However, a limit exists due to the time required to process the image data and compute the centroid of the projectile in the image. We used a minimum value of $d t=100 \mathrm{~ms}$.

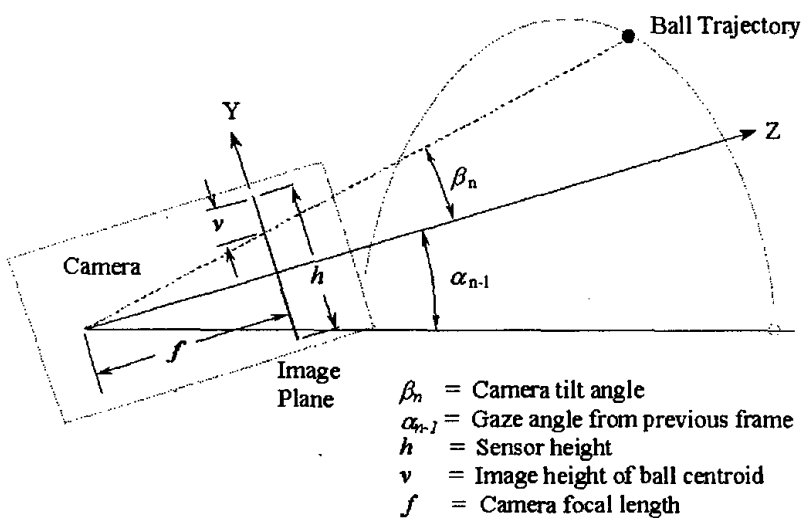

Figure 1: Task variables and architecture

\section{Motion Control Strategies}

Human fielder studies suggest the sign of $d^{2}\left(\tan \alpha_{n}\right) / d t^{2}$ can be used as an input to tell a robotic fielder whether to run forward, run back, or remain stationary. If the $\operatorname{sign}$ of $d^{2}\left(\tan \alpha_{n}\right) / d t^{2}$ is negative this indicates the projectile is beginning to drop in front of the fielder and hence the fielder needs to run forward for a successful interception. A positive sign indicates the ball is beginning to pass overhead and the fielder needs to run backward. If the value of $d^{2}\left(\tan \alpha_{n}\right) / d t^{2}$ is zero then the fielder needs to remain stationary [3]. The simple control architecture implemented for the robotic fielder is shown in Fig. 2 where the motion controller uses the sign of $d^{2}\left(\tan \alpha_{n}\right) / d t^{2}$ to help determine the fielder's next move. Although the sign of $d^{2}\left(\tan \alpha_{n}\right) / d t^{2}$ tells the fielder which direction to move, how does the fielder determine its velocity?

In McLeod's and Dienes' first experiment the human fielder started $45 \mathrm{~m}$ away from the projection point and four separate balls were launched each with a projection angle of $45^{\circ}$ but with varying initial velocities. The four balls landed at varying distances to the fielder's initial position: $-2.9 \mathrm{~m}$ behind, $+3.2 \mathrm{~m}$ in front, $+5.6 \mathrm{~m}$ in front and $+8.4 \mathrm{~m}$ in front (Fig. 3). Their second experiment launched one ball at a $45^{\circ}$ angle and a second ball at a $64^{\circ}$ angle (Fig. 3 ) with the fielder starting $45 \mathrm{~m}$ away from the projection point. The initial velocities were adjusted to ensure that both balls landed exactly $+8.5 \mathrm{~m}$ in front of the fielder. These same ball trajectories are used in the simulation experiments presented in this paper. Several different motion control strategies were simulated using these projectile conditions and are described below.

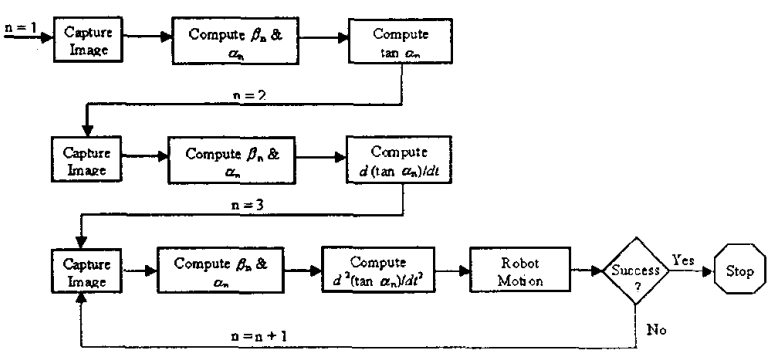

Figure 2: Robotic Fielder Control Architecture

\subsection{Motion Parameter Based Control}

McLeod and Dienes suggest that the fielder's velocity should be proportional to the magnitude of $d^{2}(\tan \alpha) / d t^{2}$. This strategy incurs difficulties when the fielder is near the ball and the gaze angle, $\alpha_{n}$, approaches its maximum value. There is an increased sensitivity of $\tan \alpha_{n}$ to $\alpha_{n}$ at the end of flight 
(a)

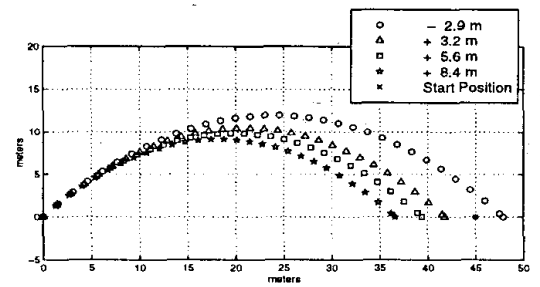

(b)

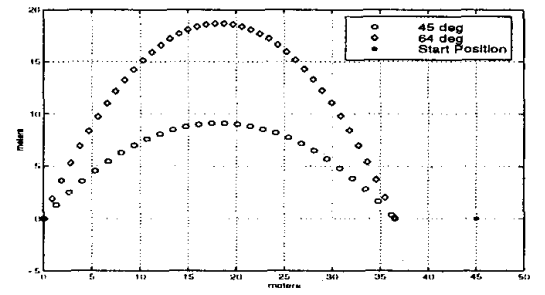

Figure 3: Ball Trajectories used. (a) Experiment 1: varying Length. (b) Experiment 2: Varying Initial Projection Angle

( $\tan \alpha_{n}$ approaches infinity as $\alpha_{n}$ approaches $90^{\circ}$ ). Also, towards the end of flight, small displacements in the fielder's position results in large changes in $\alpha_{n}$. These changes in magnitude of $d\left(\tan \alpha_{n}\right) / d t$ and $d^{2}\left(\tan \alpha_{n}\right) / d t^{2}$ result in unstable behavior of the simulated fielder. Hence, we did not consider this a viable strategy for the robotic fielder.

\subsection{Distance to Ball Strategy}

Another strategy, the omniscient fielder, assumes the fielder can judge the distance to the ball and thus would know exactly what distance to move to keep $d\left(\tan \alpha_{n}\right) / d t$ a constant (i.e. $\left.d^{2}(\tan \alpha) / d t^{2}=0\right)$. Using simulation the exact distance, $d_{n}$, between the fielder and ball are computed at each sample time as well as the value of $\alpha_{n}$. This case is studied as it demonstrates the utility of the angular measurement in determining fielder motion.

The initial value of $d\left(\tan \alpha_{2}\right) / d t$ between the first and second frames is then used to predict the exact distance the fielder needs to move to keep $d\left(\tan \alpha_{n}\right) / d t$ a constant. The desired gaze angle, $\alpha_{n}^{*}$, is given by,

$$
\alpha_{n}^{*}=\tan ^{-1}\left[\left(\frac{d\left(\tan \alpha_{2}\right)}{d t}\right) * d t+\tan \alpha_{n-1}\right]
$$

for $n>2$. The relative vertical distance, $y_{n}$, and horizontal distance, $x_{n}$, can be computed by,

$$
y_{n}=d_{n} \sin \alpha_{n}, \quad x_{n}=d_{n} \cos \alpha_{n}
$$

where $d_{n}$ is the distance between the fielder and the ball. In order to make $\alpha_{n} \approx \alpha_{n}^{*}$ the robot fielder is moved a distance, $\delta_{n}$, given by,

$$
\delta_{n}=\left(y_{n} /\left(\tan \alpha_{n}^{*}\right)\right)-x_{n}
$$

The sign of $\delta_{n}$ corresponds to the sign of $d^{2}\left(\tan \alpha_{n}\right) / d t^{2}$ and is used with the value of $d t$ to calculate the velocity of the simulated fielder between frames over the entire motion.

The first set of simulations test ball trajectories of varying length (Fig. 3). Using the distance to ball strategy the relative distance between the fielder and ball for each of the four trajectories is shown in Fig. 4 (column 1). This strategy was successful. The fielder was able to intercept all four balls indicated by the relative distance, $d_{n}$, approaching zero for all four cases. In Fig. 4(middle) the velocities for each of the four trajectories are plotted versus time. The fielder can be seen initially accelerating at his maximum rate for all four cases. For the $+8.4 \mathrm{~m}$ case the fielder accelerates during the entire motion indicating this is as far as he can run in the time allowed. Fig. 4(bottom) shows the value of $d^{2}(\tan \alpha) / d t^{2}$ for all four cases was relatively close to zero until the end of each flight. This drift is due to the lag by one frame of the calculation of $\delta_{n}$. and the fielder's subsequent motion. For the $+3.2 \mathrm{~m}$, $+5.6 \mathrm{~m}$, and $+8.4 \mathrm{~m}$ cases the negative drift indicates the ball is dropping in front of the fielder while the positive drift for the $-2.9 \mathrm{~m}$ case indicates the ball is passing overhead.

Results of the second set of simulations (projection angle varies) shows that the fielder intercepts both balls as $\delta_{n}$ approaches zero for both cases (Fig. 4 (column 2)). In Fig. 4(middle) the fielder's velocities are plotted for both trajectories. With a $45^{\circ}$ projectile angle the fielder must run (and accelerate) as fast as he can to successfully intercept the ball. For the $64^{\circ}$ projection angle the fielder accelerates at his maximum rate and then adjusts to an almost constant velocity after 1.5 seconds. If a fielder knew where to go to successfully intercept the ball they would run at a maximum speed to the exact landing point and, if allowed enough time, wait there to make the interception. This behavior was not observed in human studies [3]. The simulated fielder also does not stop but keeps running at an almost constant velocity and thus behaves in much the same way as the human fielder. As in experiment 1 , the value of $d^{2}(\tan \alpha) / d t^{2}$ stays relatively close to zero with negative drift towards the end of the flight of both balls. This indicates that the balls dropped in front of the simulated fielder.

Fig. 5 is also included to show the actual gaze angle, $\alpha_{n}$, as seen by the simulated fielder and the desired gaze angle, $\alpha_{n}^{*}$, at each sample time for the $-.2 .9 \mathrm{~m}$ and $+8.4 \mathrm{~m}$ cases. Since the value of $\alpha_{n}^{*}$ is dependent 

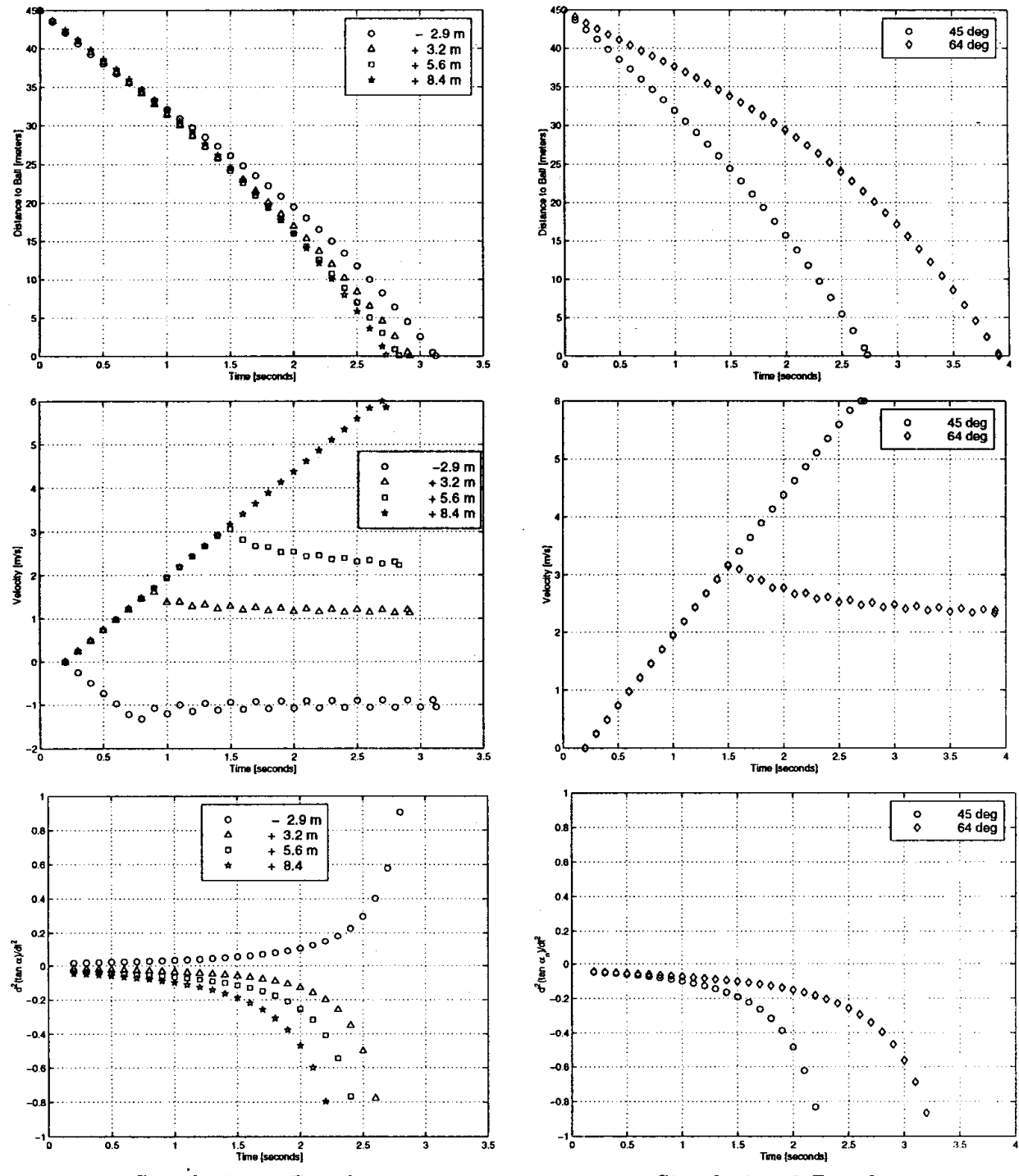

Simulation 1 Results

Simulation 2 Results

Figure 4: Simulation results for distance to ball strategy Row 1: Fielder to ball distance vs. time, Row 2: Fielder velocity vs. time, and Row $3: d^{2}\left(\tan \alpha_{n}\right) / d t^{2}$ vs. time.

on $\alpha_{n-1}$ the difference between the two angles is cumulative over the course of the motion. This did not significantly affect the strategy's success.

While the distance to ball strategy is successful and appears to closely match human studies, the goal of servoing based purely on inage information was not accomplished. Calculating the distance between the fielder and the ball makes the fielder dependent on $3-\mathrm{D}$ information. Although stereo imaging could provide $3-D$ information, the robot (and/or human) has a small baseline to which the stereo computation is notoriously sensitive. These are undesirable characteristics and probably unrealistic cues for successful human interception.

\subsection{Time to Contact Strategy}

Another strategy implements the value of the time to contact between the ball and the fielder calculated from the image size, $l_{n}$, of the ball and its first deriva- 


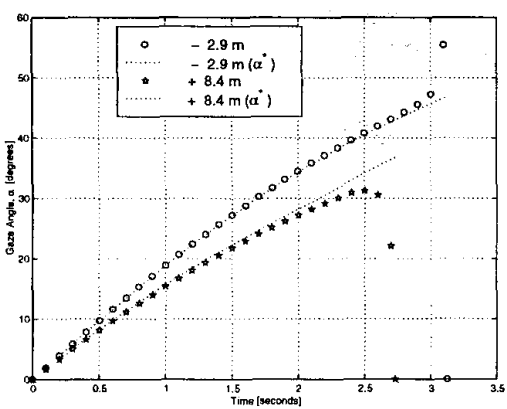

Figure 5: $\alpha_{n}$ and $\alpha_{n}^{*}$ vs. Time

tive with respect to time, $d\left(l_{n}\right) / d t$. The time to contact, $\tau_{n}$, is given by [5],

$$
\tau_{n}=l_{n} \backslash\left(d\left(l_{n}\right) / d t\right)
$$

This can be a useful parameter for many visualservoing applications where only image information is available. However, this derivation assumes that the object in the image is traveling towards the camera in a straight line with constant velocity. In Fig. 6 the value of the time to contact is computed for five different projectiles. The simulated fielder stays stationary $45 \mathrm{~m}$ from the projection point and each ball is projected at $64^{\circ}$. The initial velocity is adjusted in order that the balls will land $+8.5 \mathrm{~m}$ in front, $+4.0 \mathrm{~m}$ in front, at the fielder's position, $-4.0 \mathrm{~m}$ behind, and $-8.5 \mathrm{~m}$ behind the fielder.

Because the ball is not traveling in a straight line towards the fielder and does not have a constant velocity in the fielder's viewing frame the time to contact is not linear. The time to contact actually increases at the beginning of the flight for all five projectiles especially for the $-8.5 \mathrm{~m}$ behind projectile. If the fielder was allowed to move to intercept the ball the value of the time to contact was found to approach infinity at certain points in the flight because there was no relative change in the balls image height between frames ( $\left.d\left(l_{n}\right) / d t=0\right)$. This was especially true for relatively high projection angles.

Any attempt to use relative magnitudes of $\tau_{n}$ between successive frames as a means of velocity control for the fielder led to instabilities at points of infinite time to contact. Even by filtering these points out the fielder still did not converge on the ball using this strategy.

\subsection{Constant Acceleration Strategy}

The final control strategy developed sets the fielder's acceleration, $a$, to a constant value. The fielder then

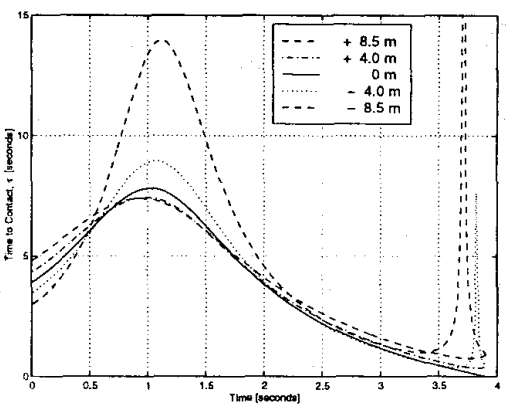

Figure 6: Time to Contact, $\tau_{n}$ vs. Time

either increases or decreases velocity depending on the sign of $d^{2}(\tan \alpha) / d t^{2}$ by a constant factor $a d t$. Using first principles the fielder can compute how fast he needs to run based purely on image information. The fielder's velocity, $v_{n}$, is given by,

$$
\begin{aligned}
& v_{n}=v_{n-1}+m_{n} a d t, \quad \text { where } \\
& m_{n}=\left\{\begin{array}{rll}
1 & \text { for } d^{2}\left(\tan \alpha_{n}\right) / d t^{2} & >0 \\
-1 & \text { for } d^{2}\left(\tan \alpha_{n}\right) / d t^{2} & <0 \\
0 & \text { for } d^{2}\left(\tan \alpha_{n}\right) / d t^{2} & =0
\end{array}\right.
\end{aligned}
$$

Using simulations with ball trajectories of varying length (Fig. 3) this strategy is shown in Fig. 7 (column 1) to be successful. The distance, $d_{n}$, between the fielder and the ball approaches zero at the end of the flight for each trajectory.

In Fig. 7(middle) the velocities for each case appear more erratic than for the distance to ball strategy given in Section 3.2. However, this appears to correspond to the more erratic velocities recorded for human fielders [3]. Fig. 7(bottom row) shows the value of $d^{2}\left(\tan \alpha_{n}\right) / d t^{2}$ for all four cases stays closer to zero longer than the values recorded using the distance to ball strategy in Section 3.2 (Fig. 4(bottom row)). However, there are substantial deviations towards the end of each flight. This is due to the inherent error caused by the control strategy itself. For the $+8.4 \mathrm{~m}$ flight the ball drops in front of the simulated fielder as indicated by the drop in $d^{2}\left(\tan \alpha_{n}\right) / d t^{2}$ at the end of the flight. The magnitude of the error at the end of the flight is given by,

$$
\delta_{\text {error }}=v_{p-1} d t+m_{p}(1 / 2) a d t^{2}
$$

where $p$ is the total number of sample periods for each flight. Only for the special case where the fielder happens to stop during the flight of the ball at the exact point where the ball will land will $\delta_{\text {error }}=0$.

Using simulations with ball trajectories of varying projection angles, $d_{n}$ approaches zero for both cases 

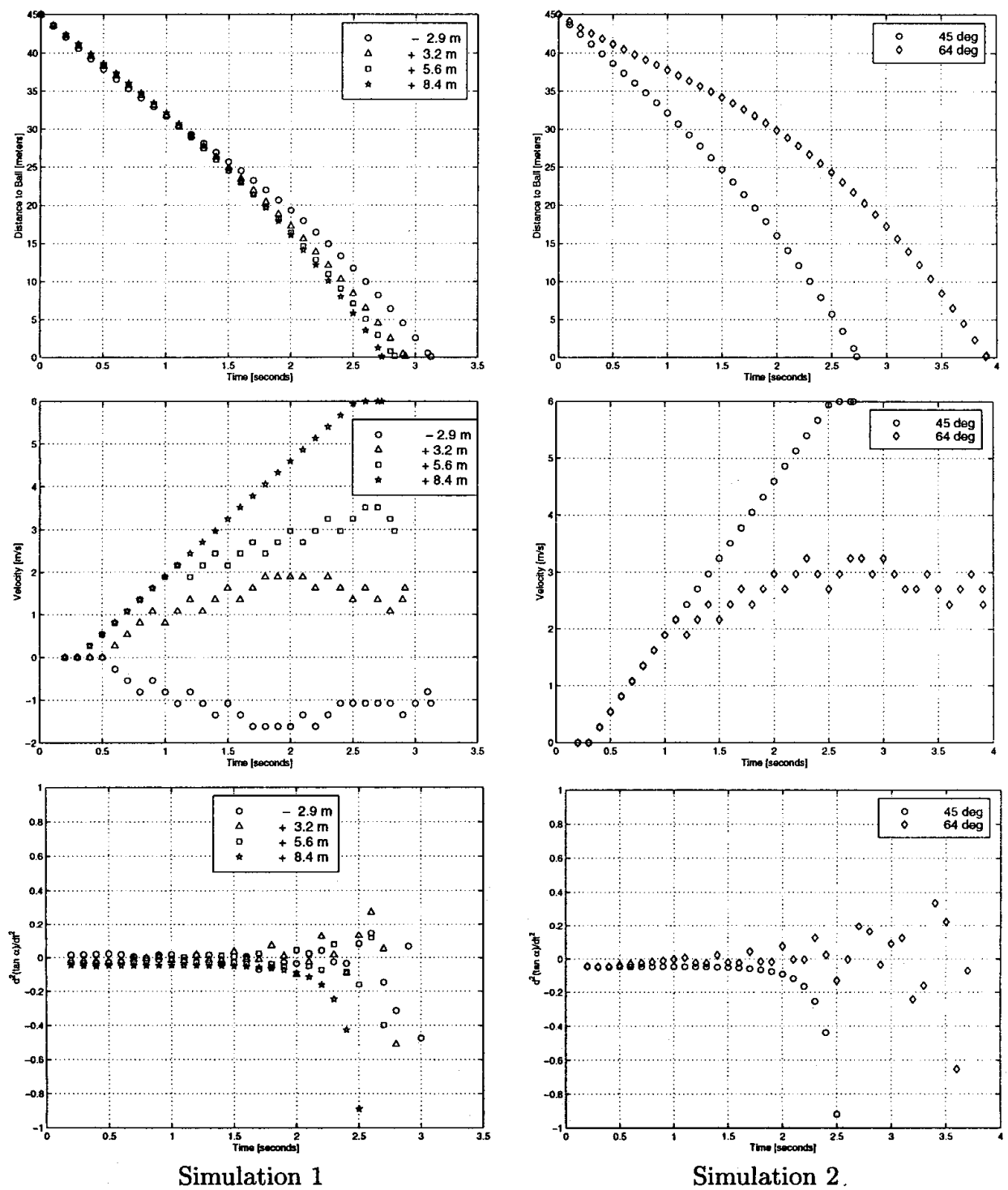

Figure 7: Simulation results for constant acceleration strategy Row 1: Fielder to ball distance vs. time, Row 2: Fielder velocity vs. time, and Row $3: d^{2}\left(\tan \alpha_{n}\right) / d t^{2}$ vs. time.

(Fig. 7 (top) ). For the ball with the $45^{\circ}$ projection angle the fielder must run as fast as he can to successfully intercept the ball (Fig. 7 , middle). For the ball with the $64^{\circ}$ projection angle the fielder accelerates at his maximum rate and then adjusts his velocity sooner than the fielder using the distance to ball strategy (Fig. 4). The fielder using a constant acceleration strategy for the $64^{\circ}$ projected ball runs more erratically than the fielder using the distance to ball strategy and appears to closely match the human results [3].
In Fig. $7\left(\right.$ bottom) the value of $d^{2}\left(\tan \alpha_{n}\right) / d t^{2}$ for both cases stay closer to zero longer than the values recorded using the distance to ball strategy (cf. Fig. 4(bottom)). Again we observe deviations towards the end of each flight with the drop in $d^{2}\left(\tan \alpha_{n}\right) / d t^{2}$ for the $45^{\circ}$ flight indicating the ball drops in front of the fielder and the sporadic values of $d^{2}\left(\tan \alpha_{n}\right) / d t^{2}$ for the $64^{\circ}$ flight indicate the ball has passed over the simulated fielder's head at the end of the flight. However, the magnitude of the errors at the ends of both 
flights are negligible (Fig. 7(top)).

Fig. 8 shows the values of the gaze angle, $\alpha_{n}$, for each of the four trajectories of varying length. The gaze angle for the ball landing the nearest $(-2.9 \mathrm{~m})$ to the fielder's initial position approaches $45^{\circ}$ which is to be expected for a parabolic flight assuming the fielder's eyes and ball's projection point are at the same height. This indicates zero error between the ball and the fielder at the time the ball landed. The final gaze angles for the balls landing further away fall short of $45^{\circ}$ which is an indication of the amount of error, $\delta_{\text {error }}$, at the time the balls land. This is to be expected since the farther the distance the simulated fielder has to cover the faster he has to run and the more error will be induced at the final positions (eq. 7). The error is negligible however compared to the initial distance between the fielder and the ball as can be seen again from the distance to ball plots in Fig. 7 .

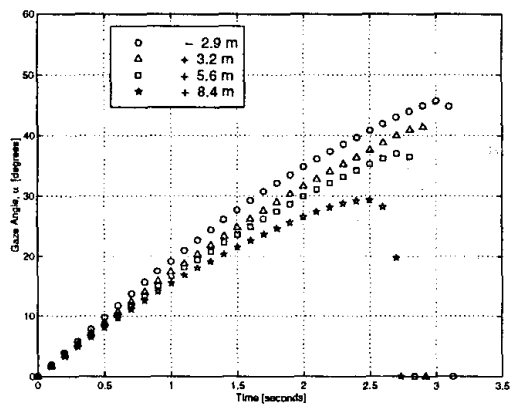

Figure 8: $\alpha_{n}$ vs. Time

The constant acceleration strategy proved to be successful in the simulations and more feasible than the distance to ball strategy because the control is purely image-based. Therefore, the constant acceleration strategy was implemented with an RWI robot to test this vision control strategy.

\section{Robot Fielder Results}

As described in Section 2 image based tracking of the ball was achieved by using a white ball against a dark backdrop and using a threshold value to determine the centroid of the ball. The robot was initially placed $3 \mathrm{~m}$ in front of the projection point of the ball with two video cameras recording the motion of the ball and robot. One video camera tape was used to determine the approximate initial projection angle of the ball while the other recorded both the ball and the robot motion together. The ball was hand-tossed by a human with varying projection angles and velocities.
The objective of the robot was to move such that the onboard camera tracking the ball would make contact with the ball at the end of the flight. For the ideal case the ball would completely fill the image at the time of interception. Twenty-four trials were recorded in all with the results of four presented here. For all trials the acceleration of the robot was set to 1200 $\mathrm{mm} / \mathrm{s}^{2}$ and with the parameters $d^{2}\left(\tan \alpha_{n}\right) / d t^{2}$ and commanded robot velocity recorded at each sample time $(d t=100 \mathrm{~ms})$.

Fig. 9 represents the data collected from Trials 4, 9,14 , and 20 . The values of the motion parameter $d^{2}\left(\tan \alpha_{n}\right) / d t^{2}$ and the corresponding commanded velocity at each point are shown. The initial projection angles determined from analysis of the videotape for Trials $4,9,14$, and 20 are $55^{\circ}, 36^{\circ}, 48^{\circ}$, and $48^{\circ}$ respectively. Also determined from analysis of the videotape was the horizontal distance traveled by each ball between the projection point and the CCD camera's initial position. These distances were approximately $300 \mathrm{~mm}, 450 \mathrm{~mm}, 250 \mathrm{~mm}$, and $100 \mathrm{~mm}$ respectively. As can be seen from the plots of $d^{2}\left(\tan \alpha_{n}\right) / d t^{2}$ (Fig. 9, column 1 ), the values varied in magnitude during the flights much more than the simulated results in Fig. 7 however, the patterns are similar. For each of the robot's results in Trials 4, 9, and 14 the ball dropped in front of the camera towards the end of the flight causing the value of $d^{2}\left(\tan \alpha_{n}\right) / d t^{2}$ to decrease. This corresponds to the simulated results for the constant acceleration strategy where the ball lands in front of the fielder at the end of the flight (i.e. $+8.4 \mathrm{~m}$ flight and $45^{\circ}$ flight in Fig. 7$)$ and the value of $d^{2}\left(\tan \alpha_{n}\right) / d t^{2}$ decreases rapidly. In Trial 20 the robot successfully intercepts the ball by making contact with the CCD camera which is indicated by the nearly zero final value of $d^{2}\left(\tan \alpha_{n}\right) / d t^{2}$.

In Fig. 9 (column 2) the commanded velocities for the robot are shown for all 4 trials. By using equation 6 the velocity was either increased or decreased at each sample time by a factor of the robot's acceleration, $a$, based on the sign of $d^{2}\left(\tan \alpha_{n}\right) / d t^{2}$.

One of the most difficult problems with the trials was the limitation of the lab environment. The human fielder results were for trials where the fielder started much farther from the projection point $(45 \mathrm{~m})$ and the flight times were much greater than could be achieved in the lab. The ball trajectories were limited by a $3.5 \mathrm{~m}$ high ceiling where the robot camera was $1.5 \mathrm{~m}$ off the floor. Additionally, the effect of the delay between commanded and actual robot velocities was magnified due to the shortened flight times (less than 1 second). The robot had limited ability to react. Optimally the trials should be run where trajectories on the scale of 

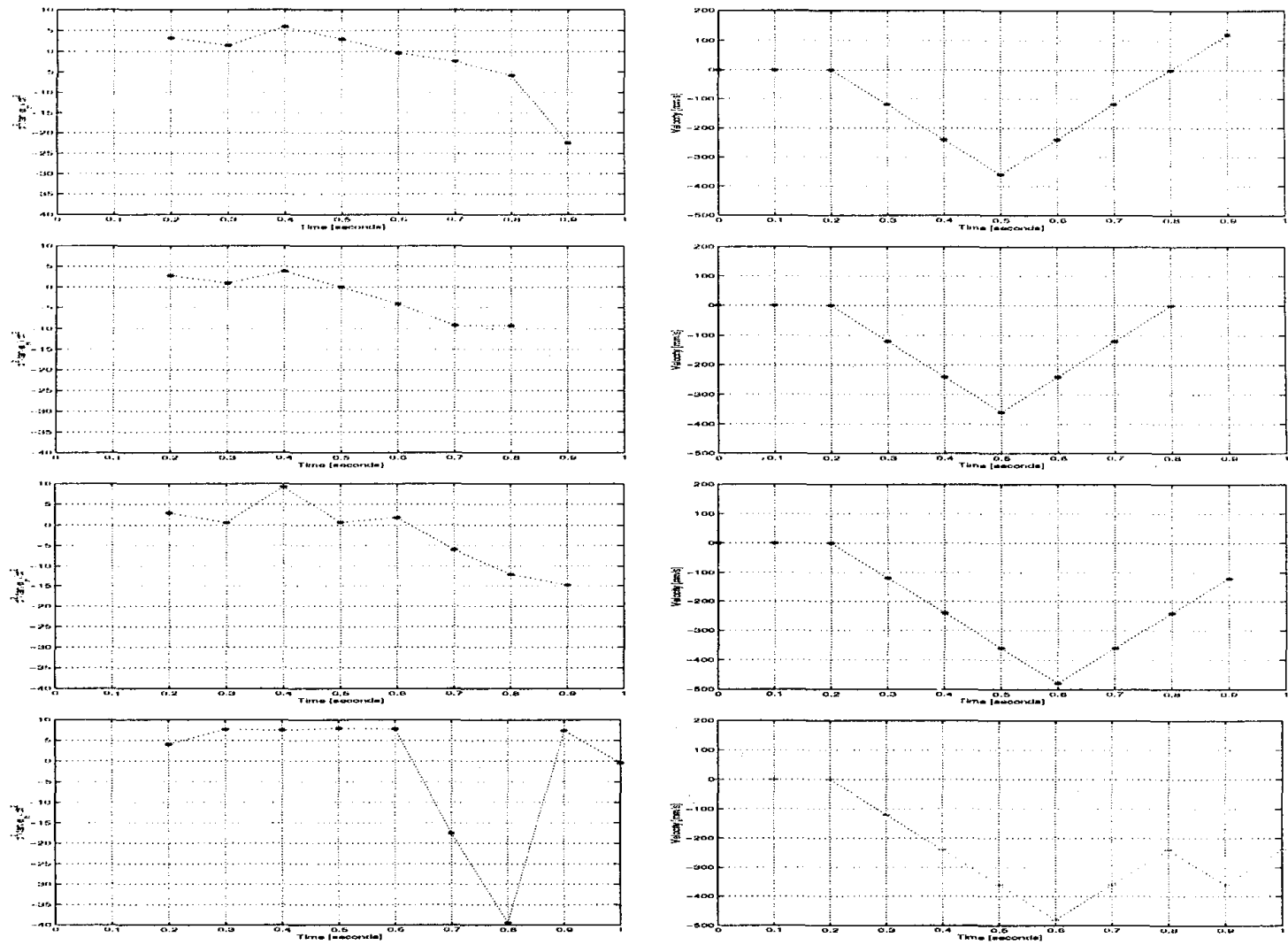

Figure 9: The left hand side shows $d^{2}\left(\tan \alpha_{n}\right) / d t^{2}$ vs. Time, the right hand side plots Fielder Velocity vs. Time. Each row shows a different trial (from top to bottom): Trial 4, Trial 9, Trial 14, Trial 20.

human fielders could be achieved, however, the trial's results show matching behavior with both the simulated and human studies [3].

\section{Conclusions}

The goal of this paper was to demonstrate that simple human vision-based approaches to visual-servoing exist and can be easily implemented. For many visualservoing tasks dynamic performance is hindered due to the need for geometrical and dynamic modeling. A successful robot motion control strategy was developed for the special case of intercepting balls undergoing projectile motion. This strategy is based on a successful human vision-based strategy which provides a much simpler image-based approach to accomplish the task. The simulated and real-world experimental results presented in this paper have shown the feasibility of this strategy. A mobile robot using this approach can successfully intercept balls with projec- tile motion and may help provide a basis for the future development of human vision-based approaches to visual-servoing.

\section{References}

[1] R.L. Andersson. A Robot Ping-Pong Player. MIT Press, 1998.

[2] P. Corke. Visual Control of Robots. Research Studies Press, 1996.

[3] P. McLeod \& Z. Dienes. How fielders intercept the ball before it hits the ground. Technical Report, Oxford and Sussex University, 1994.

[4] W.H. Press, et. al., Numerical Recipes in C. Cambridge University Press, 1988.

[5] E. Trucco \& A. Verri. Introductory Techniques for 3-D Computer Vision. Prentice-Hall, Inc., 1998. 